\title{
A estas alturas del partido
}

Un sistema de tributación debe ser lo más entendible y simple posible, para cualquier actor de la economía nacional y sobre todo, en un mundo de negocios que no sólo está dentro de las fronteras de Chile sino fuera de ella. Pero mucho más importante es que las reglas de tributación no tengan cambios de interpretación relevantes en la etapa de implementación de una reforma.

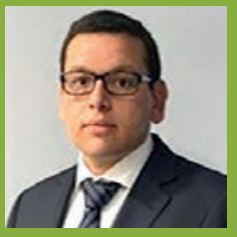

Escribe Pedro Flores Codelia Gerente Senior de EY, Contador Auditor Universidad Tecnológica Metropolitana, Diplomado en Análisis y Planificación Tributaria PUC

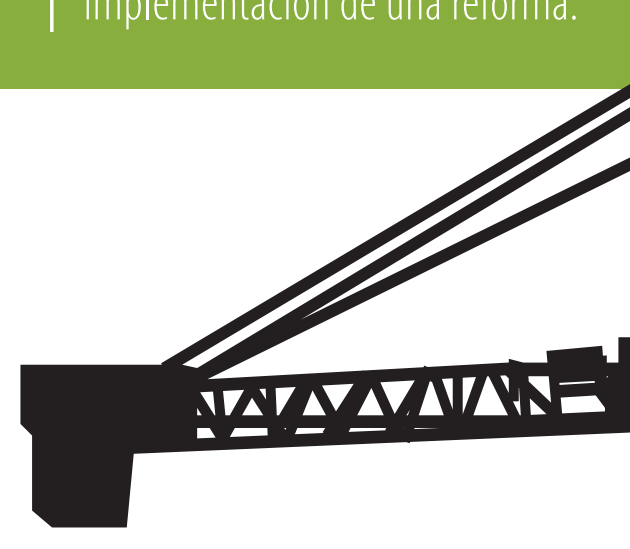

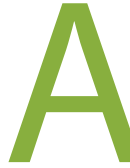

esta altura del partido -hablando en términos futbolísticos- donde los impuestos corporativos y personales han sido declarados, no es necesario profundizar si la reforma tributaria es simple, compleja, comprensible o inexplicable; puesto que ya es casi normal que cada día en la prensa escrita, redes sociales o cualquier otro medio de comunicación, expertos o entendidos en materias impositivas señalen que la implementación de esta generará mayores costos para las compañías con procesos de fiscalización largos y complejos, poniendo a prueba al regulador respecto del
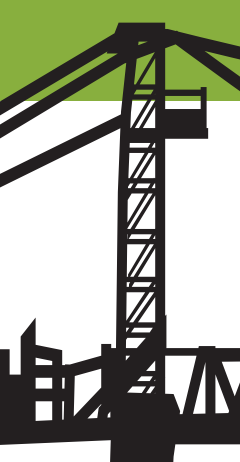

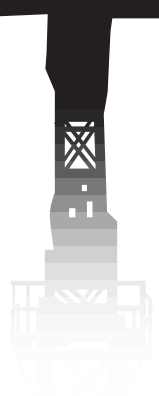

entendimiento en profundidad de lo que ha señalado a través de las instrucciones al momento de presentar las declaraciones anuales de impuestos y declaraciones juradas, que en forma directa confluyen en los impuestos personales y en los impuestos de los inversionistas extranjeros.

También ha sido público el malestar del Colegio de Contadores de Chile A.G., cuando el Servicio de Impuestos Internos (SII) adelanta las fechas de presentación de las declaraciones juradas que guardan relación directa con la declaración anual de impuestos y que -a pesar del corto plazo que el regulador entregó para su presen- tación- un $97 \%$ de los contribuyentes acogidos al régimen de la Letra $B$ del artículo 14 de la Ley sobre Impuesto a la Renta -con mucho esfuerzo y muchas inquietudes- dio cumplimiento en los plazos originales. Pero debe observarse que, en la realidad, gran parte de las declaraciones correspondientes a esta cifra será rectificada por errores en la información recibida de los agentes retenedores o-dicho honestamente- por error en la interpretación de la interpretación del SII. Por tranquilidad espiritual y como un gesto de transparencia, esperamos que el SIl declare abiertamente que un grupo importante de contribuyentes ha efectuado rectificaciones a 
sus declaraciones por los motivos señalados anteriormente.

Ahora bien, revisando uno de los temas centrales en nuestro nuevo sistema tributario, la distribución de utilidades de las compañías que optaron por el régimen(1) Semi Integrado o que por default les correspondió aplicar, tuvieron que calificar tributariamente dichas distribuciones de utilidades bajo las instrucciones del mencionado régimen y debieron asignar a tales repartos, parte del impuesto de primera categoría que las utilidades pagaron en las compañías respectivas y -de este modo- los contribuyentes finales, personas naturales o inversionistas extranjeros, utilizaron como crédito y rebajaron en general de los impuestos finales el impuesto de primera categoría asignado, pero sólo en un $65 \%$ (2). Esto es el fiel reflejo de la integración parcial del sistema tributario chileno, el cual tiene por esencia que el impuesto de primera categoría pagado por las compañías sea utilizado como crédito por los propietarios. Justamente este es el punto de discusión pública, en relación al problema de cómo reformamos la reforma sin afectar la recaudación, disminuyendo la tasa de impuesto desde un $27 \%$ a un $25 \%$ y avanzamos a un sistema de tributación totalmente integrado como era hasta el año 2016.

Para los contribuyentes que están acogidos al régimen de tributación establecido en la Letra B del artículo 14 de la Ley sobre Impuesto a la Renta, resulta más conveniente dicho régimen cuando se encuentran en un proceso de reinversión de sus utilidades en pos de mayor generación futura de beneficios a través del incremento de la inversión y con ello entregar, por la vía del pago de dividendos o retiros, los beneficios a sus propietarios como parte de la esencia de la creación de una sociedad y el aporte de capital que realizan.

Pero, entrando al área chica, las distribuciones de utilidades que las compañías realicen a sus propietarios han sido calificadas tributariamente conforme a la siguiente imputación en los nuevos registros que la reforma ha establecido, en reemplazo del Fondo de Utilidades Tributables "FUT", ellos son:

Primero, a Rentas Afectas a Impuestos (RAI), conformado por el monto total del FUT determinado al término del año 2016 más la diferencia que se genera de restar al capital propio tributario, la suma positiva del capital pagado, el saldo positivo del Fondo de Utilidades Tributables y el saldo del Fondo de Utilidades No Tributables;

Segundo, a la Diferencia de Depreciación Acelerada y Normal (DDAN), determinada por el activo fijo tributario que las compañías tuviesen;

Tercero, a Rentas Exentas (REX),

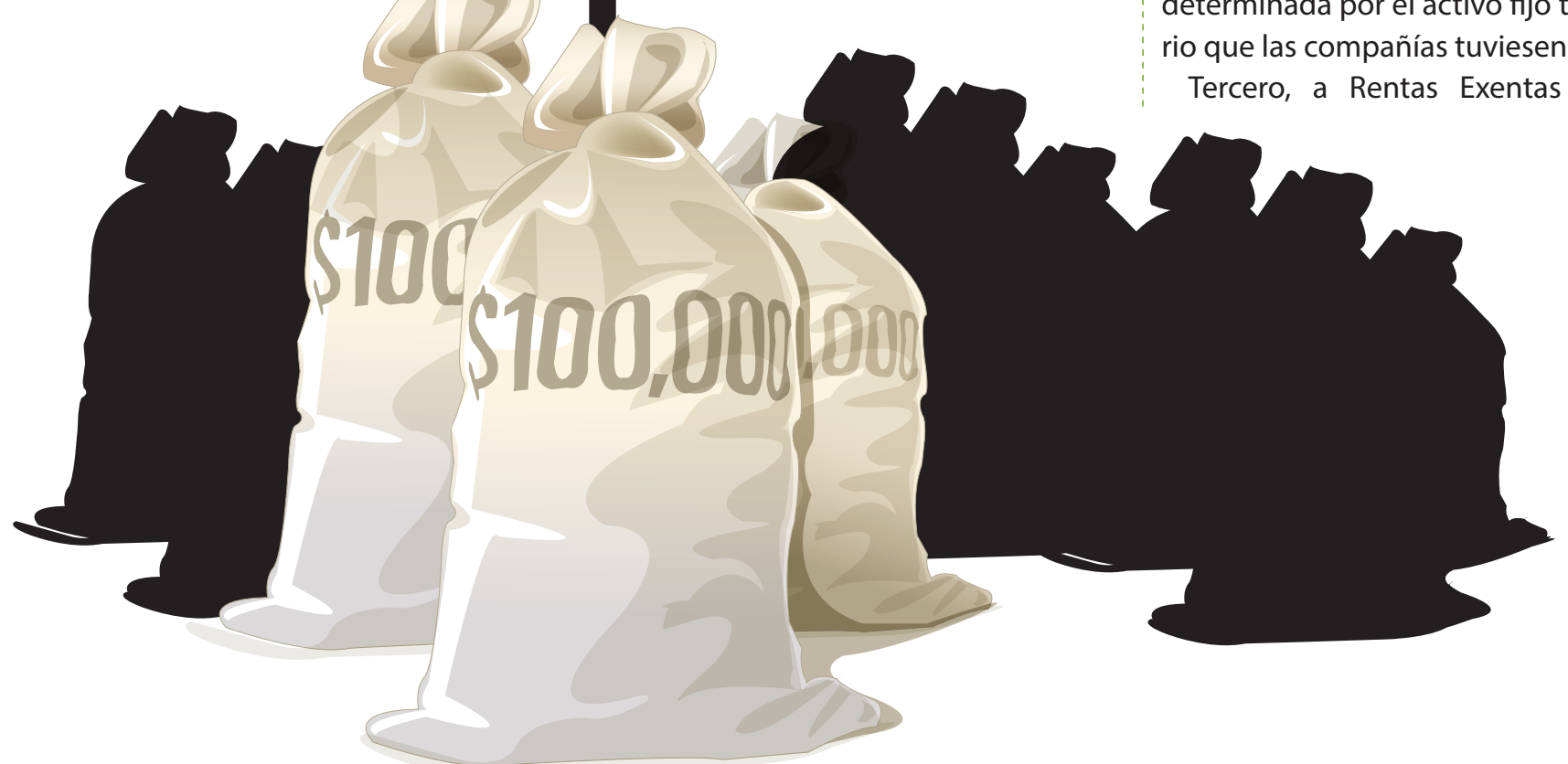


conformado por las rentas exentas e ingresos no rentas generadas en el ejercicio, más aquellas de esta misma denominación provenientes del Fondo de Utilidades No Tributables "FUNT" determinado al término del año 2016, y Cuarto, a FUT al 31 de diciembre de 2016.

En la medida que exista impuesto de primera categoría acumulado en las compañías, estos serán registrados en Saldo Acumulado de Créditos y asignados cuando las distribuciones sean imputadas en los registros RAI o DDAN o FUT Histórico.

Ahora bien, si la Ley de la Renta fuera tan clara respecto de cómo se deben asignar los créditos de primera categoría y calificar tributariamente las distribuciones de utilidades, por qué el SIl a través de Circulares y Resoluciones interpretó que sólo la diferencia positiva que resulta de restar al capital propio tributario, la suma del capital pagado, el saldo positivo del FUT y el saldo del FUNT, era parte del RAl inicial de una compañía del Régimen Semi Integrado y posteriormente, en pleno proceso de preparación de las DDJJ, específicamente en marzo de 2018, cambia de criterio a través de un Oficio, señalando que el RAl es igual al FUT, más la diferencia positiva o negativa señalada previamente, que desde una perspectiva de la declaración anual de impuestos del año 2016, fue denominado "Código 1023".

Las rentas provenientes de distribuciones de utilidades son afectadas con impuestos finales, debiendo efectuarse una declaración anual de renta -para el caso de los inversionistas nacionales- en el mes de abril del año siguiente de su percepción y en el caso de los inversionistas extranjeros en el mes de percepción. Para este último caso analicemos el impacto real de este cambio de criterio:
Una mirada desde la aplicación APLICACIÓN DE LA INTERPRETACIÓN DEL SII:

\begin{tabular}{lr} 
Antecedentes & $\mathbf{\$}$ \\
Capital propio tributario & 500 \\
FUT & 100 \\
FUNT & 150 \\
Capital pagado & 400 \\
Código 1023 & $(150)$ \\
Crédito de primera categoría & 25,5 \\
\hline Distribución de utilidades & 150
\end{tabular}

\begin{tabular}{lrrrr} 
Registro de rentas & RAI & REX & SAC & FUT \\
Saldo de inicio & 100 & 150 & 25,5 & 100 \\
$\begin{array}{l}\text { Menos: } \\
\text { Distribución }\end{array}$ & $(100)$ & $(50)$ & $(25,5)$ & \\
\hline Saldo & 0 & 100 & 0 & 100
\end{tabular}

Cálculo de impuestos

\$

\begin{tabular}{lr} 
Distribución & 100 \\
Incremento & 25,5 \\
\cline { 2 - 2 } Total & 126 \\
\hline Impuesto adicional 35\% & 44 \\
Crédito de primera & $(25,5)$ \\
\cline { 2 - 2 } Total impuesto & 18,4
\end{tabular}

APLICACIÓN DE LA LEY Y OFICIO

\begin{tabular}{lrrrr} 
Registro de rentas & RAI & REX & SAC & FUT \\
Saldo de inicio & 0 & 100 & 25,5 & 100 \\
$\begin{array}{l}\text { Menos: } \\
\text { Distribución }\end{array}$ & 0 & $(100)$ & $(25,5)$ & $(50)$ \\
\hline Saldo & 0 & 0 & 0 & 50
\end{tabular}

Cálculo de impuestos \$

\begin{tabular}{lr} 
Distribución & 50 \\
\hline Incremento & 12,8 \\
\cline { 2 - 2 } & 63 \\
\hline Total & 22 \\
Impuesto adicional 35\% & $(12,8)$ \\
\hline Crédito de primera & 9,2 \\
\hline Total impuesto
\end{tabular}

(1) Régimen establecido en el artículo 14 Letra B de la Ley de Impuesto a la Renta vigente a contar del 01 de enero de 2017.

(2) Este porcentaje será de un $100 \%$ en la medida que el inversionista extranjero sea residente en un país con el cual Chile tenga con Convenio para Evitar la Doble Tributación o se encuentre suscrito en período de ratificación hasta el año 2022. 


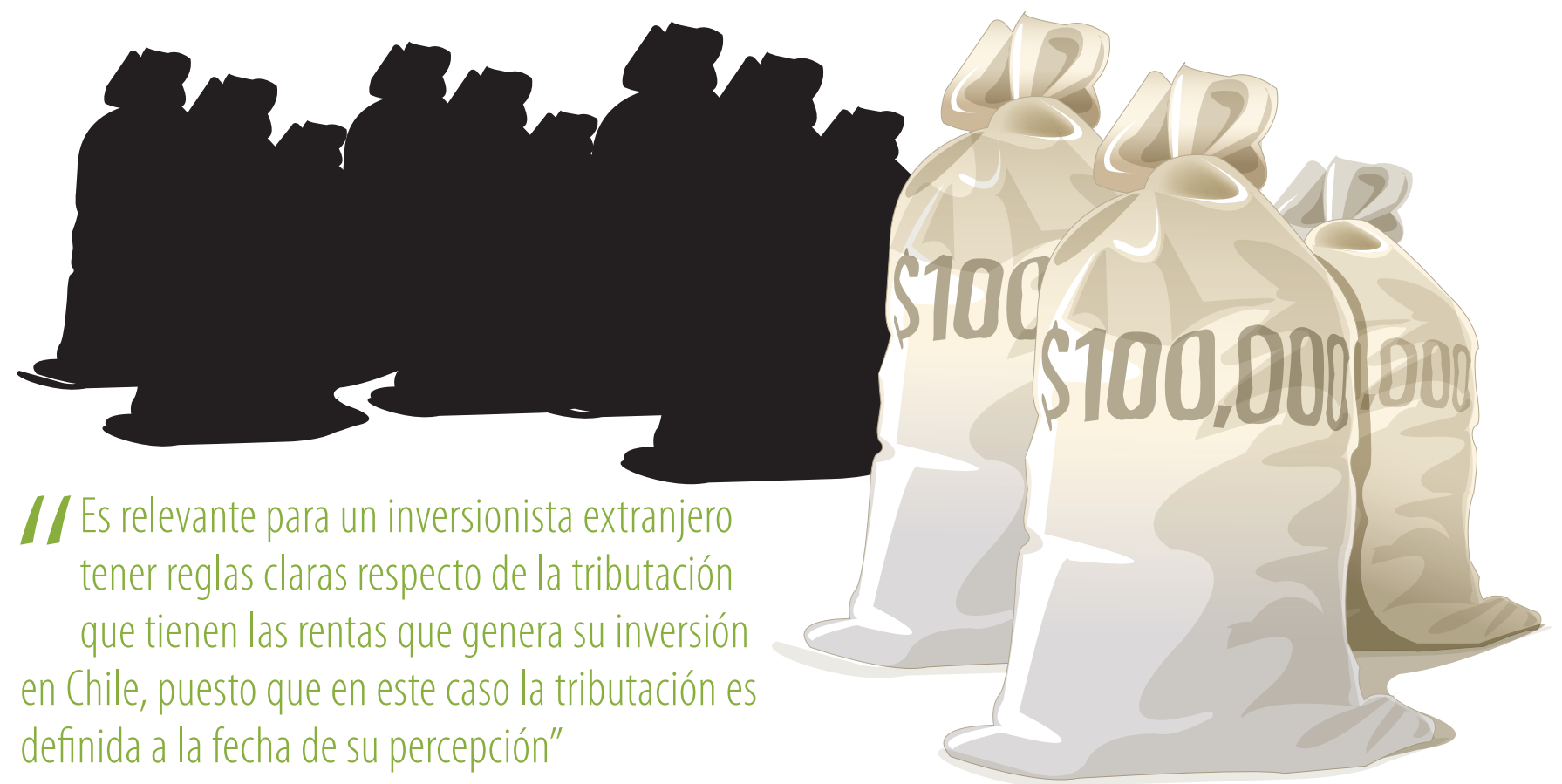

Como es evidente, la aplicación del criterio de imputación de utilidades establecido en la Ley de la Renta, en contraposición a la interpretación que el regulador entregó a las compañías durante el año 2016, generaría para las arcas fiscales una menor recaudación por las devoluciones de impuestos en beneficio de aquellos inversionistas extranjeros en compañías na- cionales que se encuentren en el ejemplo analizado, puesto que las distribuciones deberían haber sido calificadas tributariamente como Rentas Exentas y -por tanto- no tributar con uno de los impuestos finales como es el caso del Impuesto Adicional a la Renta.

Es relevante para un inversionista extranjero tener reglas claras respecto de la tributación que tienen las rentas que genera su inversión en Chile, puesto que en este caso la tributación es definida a la fecha de su percepción. Las consecuencias de esta reconsideración por parte del regulador no serán más que solicitudes de devoluciones de impuestos pagados en exceso, con un costo para los inversionistas en la recuperación de impuestos en los tiempos que el regulador considere pertinente.

\section{UNA MIRADA HACIA EL FUTURO}

Más que dar ejemplos respecto de cómo la implementación de la reforma tributaria ha generado complejidades en el proceso de declaración de impuestos anuales del año 2017, tanto para las compañías chilenas como para sus propietarios, es necesario dar una mirada más integral a este proceso de discusión de simplificar la reforma (dicho sea de paso, debe ser una nueva reforma) y cada actor que interviene en estas materias debiese aportar a este debate y proponer ideas que tiendan a:

-Generar un sistema tributario simple y entendible para cada agente que interviene en la economía en Chile.

-Proponer la reducción de información que debe entregarse al regulador, a través del proceso de declaraciones juradas, si gran parte de ella ya la tiene en sus sistemas vía el Registro de Compra y Ventas, emisión de boletas de honorarios electrónicas, y contabilidad electrónica, pero al mismo tiempo, impulsar fuertemente este último sistema de información.

-Fiscalizaciones focalizadas en los riesgos de la industria más que excesivas solicitudes de documentos que se produce en el transcurso de estos procesos, cuando ya están disponibles en los medios electrónicos que el mismo regulador ha puesto a disposición de las compañías.

-Mayor interacción con el mercado para un mayor entendimiento de los negocios de las distintas industrias y el impacto de las interpretaciones efectuadas por el SIl y así aplicar reglas claras pero que al mismo tiempo sean apropiadas para la industria. Un ejemplo claro es la aplicación del régimen de tributación del 14 B a los Fondos de Inversiones y Fondos Mutuos, y

-No lograremos ganar el partido, si junto con las grandes modificaciones que la nueva Administración ha señalado -como son la reducción de la tasa de impuesto de primera categoría de un $27 \%$ a un $25 \%$ y la integración total del sistema tributario- no se avanza en tener un sistema tributario simple e integrado, con un organismo regulador conversando con los agentes económicos para emitir normas que sean acordes a los negocios en Chile y el extranjero, utilizando con mayor fuerza la tecnología para facilitar el cumplimiento tributario de las compañías y sus propietarios. 